\section{The attentional blink: Increasing target salience provides no evidence for resource depletion. A commentary on Dux, Asplund, and Marois (2008)}

\author{
Christian N. L. Olivers \\ Vrije Universiteit Amsterdam, Amsterdam, The Netherlands
}

ThOMAS M. SPALEK

Simon Fraser University, Vancouver, Canada

JUN-ICHIRO KAWAHARA

National Institute of Advanced Industrial Science and Technology, Tsukuba, Japan

AND

VINCENT Di LOLLO

Simon Fraser University, Vancouver, Canada

The authors have argued elsewhere that the attentional blink (AB; i.e., reduced target detection shortly after presentation of an earlier target) arises from blocked or disrupted perceptual input in response to distractors presented between the targets. When targets replace the intervening distractors, so that three targets (T1, T2, and T3) are presented sequentially, performance on T2 and T3 improves. Dux, Asplund, and Marois (2008) argued that $T 3$ performance improves at the expense of T1, and thus provides evidence for resource depletion. They showed that when T1 is made more salient (and presumably draws more resources), an $A B$ for $T 3$ appears to reemerge. These findings can be better explained, however, by (1) the relationship between T1 and T2 (not T1 and T3) and (2) differential salience for T3 in the long-lag condition of Dux et al.'s study. In conclusion, the Dux et al. study does not present a severe challenge to input control theories of the $A B$.

There is no denying that the resources available to our cognitive system are limited. But does the attentional blink (AB) phenomenon provide evidence for such resource limitations? Dux, Asplund, and Marois (2008) claimed that it does. We argue that their findings provide no basis for such a claim.

The $\mathrm{AB}$ is the finding that the second of two targets is often missed when both appear amidst a stream of distractors displayed in rapid serial visual presentation (RSVP) (Raymond, Shapiro, \& Arnell, 1992). Theoretical accounts lay the cause on a limited-capacity processing stage, or bottleneck, relatively late in the information-processing sequence. Processing of the first target (T1) is said to deplete vital mental resources, to the detriment of the second (T2). There are several versions of this theory (e.g., Chun \& Potter, 1995; Jolicœur \& Dell'Acqua, 1998), but all share the idea that T2 is starved for resources, and that $\mathrm{T} 1$ is the culprit.
A recent set of studies has posed a severe challenge to resource depletion accounts (Di Lollo, Kawahara, Ghorashi, \& Enns, 2005; Olivers, van der Stigchel, \& Hulleman, 2007; see also Kawahara, Kumada, \& Di Lollo, 2006). The critical finding in these studies was the absence of an $\mathrm{AB}$ deficit for the third of three targets presented sequentially. We refer to this as the TTT or uniform condition, because the three sequential items belong to the same (target) category. In contrast, a substantial AB deficit is found when two targets are separated by a single distractor. We refer to this as the TDT or varied condition. The absence of an $\mathrm{AB}$ in the uniform/TTT condition is problematic for resource depletion accounts because they predict that having to process an extra target should result in even more depletion, and thus a more severe AB. More generally, the lack of an AB in the uniform/TTT condition puts in question the claim that $\mathrm{T} 1$ processing is the direct cause of the $\mathrm{AB}$, because $\mathrm{T} 1$ is processed in both the varied/TDT and uniform/TTT conditions, yet an AB occurs in one but not in the other. Instead, the evidence points to the post-T1 distractor, not $\mathrm{T} 1$ itself, as the cause of the AB.

Di Lollo et al. (2005) and Olivers and colleagues (Olivers, 2007; Olivers et al., 2007) have proposed that the problem is not so much one of attentional resources, but of attentional control. They assume that observers implement an input filter or attentional set aimed at selecting targets and ignoring distractors. Performance for T1 is usually accurate, because $\mathrm{T} 1$ matches the attentional set. The two accounts from these studies differ somewhat on the exact role of the post-T1 distractor and its effect on T2. Di Lollo et al. proposed that the distractor disrupts or resets the input filter, so that subsequent targets are no longer selected. Olivers and colleagues proposed that the post-T1 distractor is enhanced by the excitatory attentional response evoked by $\mathrm{T} 1$, which in turn triggers a strong inhibitory response that affects subsequent targets. Whether the post-T1 distractor disrupts or inhibits the input, both accounts agree that the $\mathrm{AB}$ derives from a selection, not a resource, deficit.

\section{Resource Depletion Back in the Saddle?}

Dux et al. argued in favor of a resource depletion account by noting that, in the white baseline condition of their Experiment 1, final-target performance was higher and T1 performance lower in the uniform/TTT condition than in the varied/TDT condition. The same result was found by Di Lollo et al. (2005) and Olivers and colleagues (Olivers, 2007; Olivers et al., 2007) in their original studies. Dux et al. regarded this result as evidence for a direct trade-off between $\mathrm{T} 1$ and the final target: When more resources are absorbed by $\mathrm{T} 1$, the final target is starved, and an $\mathrm{AB}$ follows. Thus, they claimed that the $\mathrm{AB}$ was absent in the uniform/TTT condition because not enough 
resources were deployed to $\mathrm{T} 1$, leading to their prediction that if $\mathrm{T} 1$ could be made to attract more resources, the ensuing targets would be starved, and an $\mathrm{AB}$ for the final target would be in evidence in the uniform/TTT condition. They achieved this by presenting red targets after a stream of white distractors, as compared with presentation of an all-white stream. It was reasoned that a red T1 after a white distractor would be more salient, and thus draw more resources, to the detriment of the ensuing targets.

In their Experiment 2, Dux et al. argued that the results of Experiment 1 were indeed an instance of the $A B$, as evidenced by the finding that in the uniform/TTT condition, the deficit for the final target disappeared when it was presented at a longer lag after T2; after all, a lag effect has been the typical diagnostic of an AB. Dux et al. concluded (p. 813) that "a T1 processing bottleneck limits our ability to consciously perceive information that is distributed across time." In the present study, we claim that this conclusion is not warranted, and that Dux et al.'s study provides no direct evidence for resource depletion. We support these claims with empirical findings showing that other mechanisms can adequately explain their resultsmechanisms that are fully consistent with control-based accounts, without recourse to resource depletion.

\section{EXPERIMENT 1 \\ No Trade-Off Between the First and Last Targets}

Dux et al. rightly pointed out that the improvement for the final target in the uniform/TTT conditions relative to the varied/TDT conditions in Di Lollo et al. (2005) and Olivers et al. (2007) was accompanied by decrements for $\mathrm{T} 1$ - and then they demonstrated the same effect once again. Does this mean that the final target caused performance on T1 to go down? This is indeed what a resource depletion account predicts. Targets act like communicating vessels: What one gains in resources, the others lose.

However, it is essential to realize that all of these experiments employed three targets. If they act as communicating vessels, there would be three here, not just two. In the present experiment, we show that, contrary to Dux et al.'s assertion, the deterioration of $\mathrm{T} 1$ in the three-target condition is due to the second target, at lag 1 , not to the third target, at lag 2.

\section{Method}

We used the data from the two- and three-target conditions of Olivers et al. (2007), in which either digit or letter targets were inserted in a stream of either letters (Experiment 1) or fantasy characters (Experiment 2); a total of 24 participants took part in the experiments. All items were black on a gray background. Further details can be found in Olivers et al.'s study. Specifically, we compared four conditions: TT, TDT, TTT, and TDTT, with each T denoting a target and each $D$ a distractor. We collapsed the results across experiments for maximum power ( $N=24$; for the crucial comparisons, there were no interactions with experiment). We performed the analyses as in Olivers et al.'s study, including a correction for guessing. ${ }^{1}$

\section{Results and Discussion}

The results for T1 are illustrated in Figure 1. As Olivers et al. (2007) reported, T3 performance was comparable to

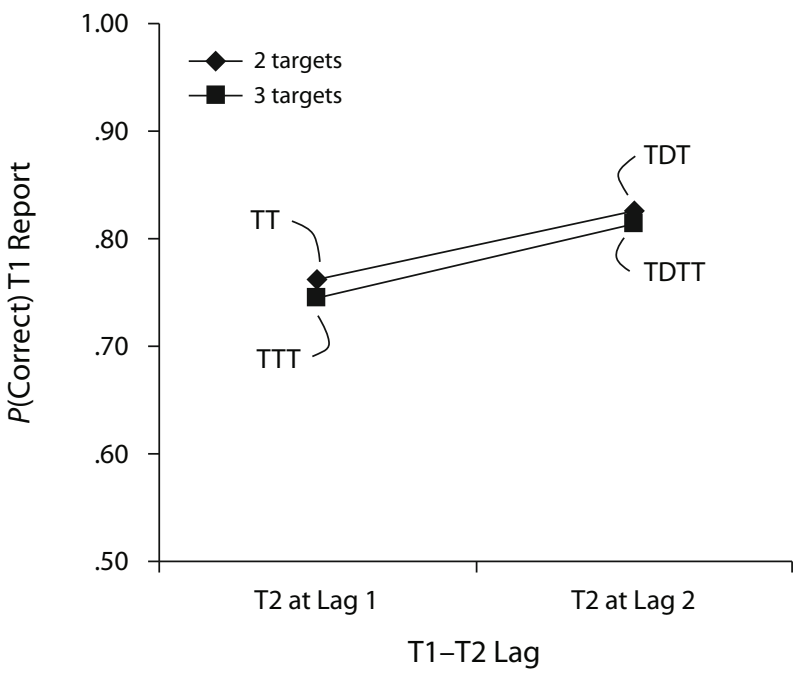

Figure 1. T1 report accuracy for two- and three-target conditions in Experiment 1, as a function of T1-T2 lag. For clarification, the conditions have been labeled with the corresponding target/distractor sequences $(\mathrm{T}=$ target, $\mathrm{D}=$ distractor $)$.

that for T1. A repeated measures ANOVA with number of targets ( 2 or 3$)$ and T2 lag ( 1 or 2$)$ as factors revealed only a main effect of lag $\left[F(1,23)=12.58, M S_{\mathrm{e}}=.008, p<\right.$ .002 ; all other $F \mathrm{~s}<1$ ]. The results are clear: T1 accuracy deteriorated when another target was presented at the lag 1 position; adding a third target had no effect whatsoever on T1. Kawahara et al. (2006) found the same result, that T1 performance was bad when $\mathrm{T} 1$ was immediately followed by a T2. In contrast, neither the presence nor the relative temporal position of T3 had an effect on T1. This strongly suggests that the T1-T3 trade-off alluded to by Dux et al. is in fact a T1-T2 trade-off.

The general lesson to be learned here is that a single experimental manipulation may have two independent effects. Experimental manipulation X may cause both effect $A$ and effect $B$, so that $A$ and $B$ will covary, but by no means does this indicate that $A$ causes $B$. Inserting another target between the first and final targets causes improvements for the final and deterioration for the first target, but this does not mean that the improvement of the final target causes the deterioration of the first (or vice versa). Dux et al. misinterpreted a correlation as a causal relationship.

\section{EXPERIMENT 2 \\ Relative Salience, Not Resource Depletion, Determines Performance}

Dux et al. performed a second experiment aimed at supporting the hypothesis that the T3 deficit observed under uniform conditions in their Experiment 1 was indeed an $\mathrm{AB}$. To this end, they inserted three red targets in a stream of white distractors. There were two conditions: In the short-lag condition, the three targets were presented sequentially; in the long-lag condition, five white distractors were inserted between T2 and T3. The two display se- 

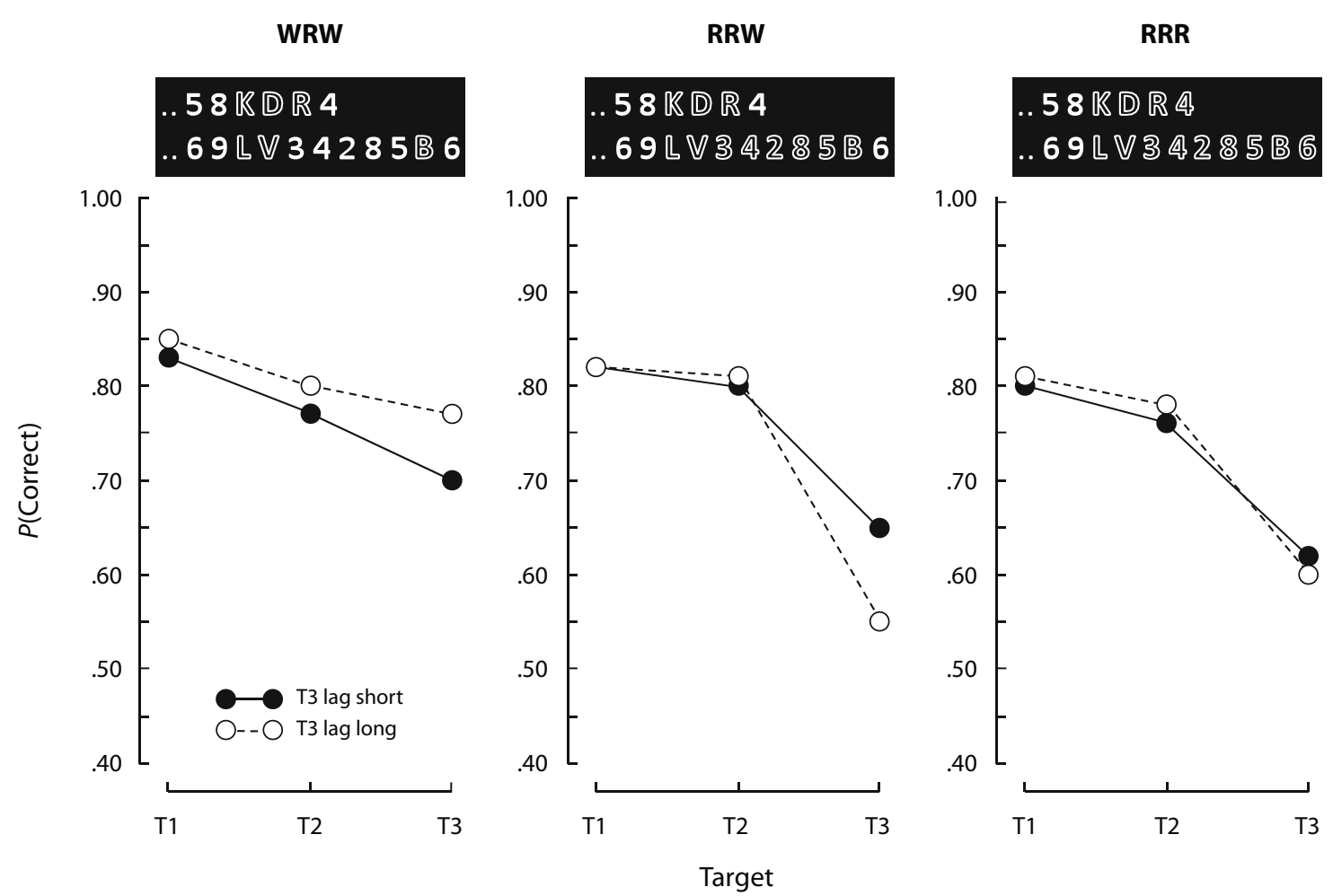

Figure 2. Report accuracy for T1, T2, and T3 as a function of lag (short or long) and relative T3 salience (WRW, RRW, and RRR conditions; the middle $R$ denotes the red T3, and the flanking $W \mathrm{~s}$ or $R$ s denote white or red distractors preceding or following it at long lags). At the top of each panel is a schematic representation of the display sequence in each of the three conditions (upper row, short T1-T3 lag; lower row, long T1-T3 lag). The background is black (as in the actual displays); white items are rendered as white, and red items are rendered as outline characters.

quences were as illustrated at the top of the leftmost panel of Figure 2. Dux et al.'s finding that the accuracy of T3 performance was higher at the longer lag was interpreted as confirming that the $\mathrm{T} 3$ deficit was indeed an $\mathrm{AB}$.

What Dux et al. did not consider was that the onset of the red T3 stood out more in the long-lag condition-in which it was preceded by a string of white items - than in the short-lag condition - in which it was preceded by other red items. As any theory of attention would predict, the more salient candidate stands a better chance of being detected. In the present work, we tested the hypothesis that in Dux et al.'s Experiment 2, T3 performance was more accurate at the longer than at the shorter lag not because it had recovered from the $\mathrm{AB}$, but simply because it was more salient.

As illustrated at the top of Figure 2, we employed three short-lag conditions in which three red targets were presented sequentially, as well as three long-lag conditions in which five distractors were inserted between $\mathrm{T} 2$ and T3, as follows: white-red-white, red-red-white, and redred-red (WRW, RRW, and RRR, respectively, with the middle R denoting the red T3, and the flanking Ws or Rs denoting white or red distractors preceding or following it). Following the same argument that Dux et al. made for T1, the onset of T3 was assumed to be more salient in condition WRW, in which it was preceded by white items, than in the other two conditions, in which it was preceded by red items. If, as Dux et al. claimed, the critical factor in T3 performance is the lag, T3 should be reported more accurately at the longer than at the shorter lag in all conditions. If, however, the critical factor is salience, T3 accuracy should be higher at the longer than at the shorter lag in condition WRW, but not in the other two conditions.

\section{Method}

Participants. Twenty-four undergraduate students at Simon Fraser University participated in Experiment 2 for course credit or payment. All reported normal or corrected-to-normal vision.

Stimuli. The stimuli were presented in the center of a black screen ( $R G B=0,0,0 ; 2.5 \mathrm{~cd} / \mathrm{m}^{2}$ ) in Courier New font and subtended approximately $0.7^{\circ}$ vertically at a viewing distance of approximately $57 \mathrm{~cm}$; they were either red $\left(\mathrm{RGB}=255,0,0 ; 22.0 \mathrm{~cd} / \mathrm{m}^{2}\right.$ ) or white $\left(\mathrm{RGB}=110,110,110 ; 22.0 \mathrm{~cd} / \mathrm{m}^{2}\right)$. The red and the white items were equated for luminance, in order to preclude differential luminance masking effects between the two colors. ${ }^{2}$ The targets were three red uppercase letters drawn from the English alphabet, excluding I, O, Q, and Z. The distractors were digits ranging from 2 to 9 , selected randomly, with the constraint that the selected digit had not occurred in one of the two preceding items.

Design. There were three independent variables: T3 lag, T3flanking colors, and target position. T3 lag was either short (T1, T2, and $\mathrm{T} 3$ presented sequentially, as illustrated in the upper row of the insets at the top of Figure 2) or long (T1 and T2 presented sequentially, followed by five distractors before T3, as illustrated in the lower row of the insets at the top of Figure 2). As for the T3-flanking colors, the distractors preceding and following T3 in the long-lag condition were as illustrated in the insets at the top of Figure 2 and 
as described above. Target position was defined by ordinal sequence: $\mathrm{T} 1, \mathrm{~T} 2$, or $\mathrm{T} 3$.

Procedure. The experiment consisted of three blocks of trials. The WRW, RRW, and RRR conditions were run in separate blocks, with order counterbalanced across observers. On any given trial, the T3 lag could be either short or long, with the constraint that each block contained equal numbers of short and long lags. At the beginning of each trial, a white fixation cross $\left(0.3^{\circ}\right)$ was presented in the center of the screen and remained on view until the observer initiated the trial by pressing the space bar. At the end of each trial, observers were required to identify the three target letters by pressing the corresponding keys on the keyboard. Each item in the RSVP stream was displayed for $100 \mathrm{msec}$, without any temporal gap between successive items. The RSVP stream began with between 5 and 10 white digits, determined randomly on each trial, and ended with a single distractor after T3. There were 80 trials per block, 40 at each lag, for a total of 240 trials.

\section{Results and Discussion}

The results, illustrated in the bottom panels of Figure 2 , were analyzed in a 3 (condition: WRW, RRW, or RRR) $\times 2$ (lag: short or long) $\times 3$ (target: T1, T2, or T3) ANOVA. The analysis revealed significant effects of condition $\left[F(2,46)=3.83, M S_{\mathrm{e}}=.035, p<.029\right]$ and target $\left[F(2,46)=25.03, M S_{\mathrm{e}}=.049, p<.001\right]$. There were also three significant interaction effects: condition $\times$ lag $\left[F(2,46)=6.98, M S_{\mathrm{e}}=.006, p=.002\right]$, condition $\times$ target $\left[F(4,92)=10.10, M S_{\mathrm{e}}=.008, p<.001\right]$, and condition $\times$ lag $\times$ target $\left[F(4,92)=5.74, M S_{\mathrm{e}}=.004, p<\right.$ $.001]$. The significant three-way interaction was examined further by performing statistical analyses similar to those performed in Dux et al.'s Experiment 2, separately for each condition.

The pattern of results in condition WRW (Figure 2) replicated the findings of Dux et al. (Experiment 2). For this reason, all statistical analyses performed on condition WRW were one-tailed tests. Performance was significantly lower for $\mathrm{T} 3$ than for $\mathrm{T} 1$ at both the short lag $[t(23)=4.17, p<.001]$ and the long lag $[t(23)=3.89$, $p<.001]$. The T3 impairment relative to T1 was greater when the lag was short $[t(23)=1.92, p=.034]$. It is worth noting that this difference was also highly significant when the stimuli were not isoluminant (see note 2), as had been the case in Dux et al.'s study $[t(23)=4.07$, $p<.001]$.

The pattern of results in conditions RRW and RRR, however, differed markedly from that in condition WRW. As was the case for condition WRW, performance in conditions RRW and RRR was significantly lower for T3 than for T1 at both the short and long lags: RRW (short lag), $t(23)=4.19, p<.001$; RRW (long lag), $t(23)=5.46$, $p<.001$; RRR (short lag), $t(23)=3.94, p=.001$; RRR (long lag), $t(23)=5.50, p<.001$. For the purposes of the present work, however, the important finding was that the T1-T3 difference was smaller for the long lag in neither condition RRW nor RRR (see Figure 2). Indeed, whereas the difference was not significant in condition RRR, it was actually in the opposite direction in condition RRW $[t(23)=2.11, p=.046]$. Thus, the results reported by Dux et al. were replicated in the present experiment in condition WRW - in which T3 stood out from the surrounding items - but not in conditions RRW and RRR- in which the red T3 did not stand out from the leading red distractors.

These results show that with equal $\mathrm{T} 1$ performance (and thus, according to Dux et al.'s logic, equal demands in terms of resources), one can measure quite different performance levels for T3, depending on the type of items that precede or follow it. In other words, T3 performance depends on relative salience, not on differential resource depletion. The present WRW condition replicated the Dux et al. results: When T3 salience was higher at the long than at the short lag, T3 accuracy was also higher at the long lag. When salience was equated, however, as in the RRR condition, the superior T3 performance disappeared, thus disconfirming predictions based on resource depletion. This strongly suggests that the superior T3 performance at a long lag found in Dux et al.'s Experiment 2 was due not to recovery from the $\mathrm{AB}$, but to the enhanced salience of T3.

The importance of relative salience was further corroborated in our condition RRW, in which performance for T3 was actually worse at the long than at the short lag. This makes sense, because after a series of red items, the final white distractor becomes more salient, and is therefore more likely to interfere with $\mathrm{T} 3$ processing. Likewise, there is no reason to assume that relative salience would not have played an important role even in the short-lag conditions of Dux et al.'s experiments (including their Experiment 1): A red T3 following two red items simply stands out less than a red T1 following white items. In the General Discussion, we consider why accuracy for a red T3 may even be lower than for a white T3.

\section{GENERAL DISCUSSION}

The $\mathrm{AB}$ demonstrates that having to process a leading target causes the processing of a trailing target to be impaired when the latter target is presented shortly after the first. The crucial question is whether this effect occurs because of mechanisms akin to communicating vessels: If one goes up, the other must automatically come down. Dux et al. sought to provide support for this idea. Here, we have shown that the ostensibly communicating vessels may in reality be disconnected from one another.

Experiment 1 showed that the trade-off between $\mathrm{T} 1$ and T3 in uniform/TTT sequences turns out to be a trade-off between T1 and T2 (when T2 is presented at lag 1). Such T1-T2 trade-offs have been reported in the literature (Potter, Staub, \& O'Connor, 2002) and do not explain why an immediately preceding T2 causes the relative sparing of T3. Experiment 2 showed that an improvement in T3 performance at a long lag, which Dux et al. attributed to recovery from the $\mathrm{AB}$, was actually due to enhanced discriminability of the T3 stimulus. Thus, what Dux et al. attributed to an internal factor (recovery from resource depletion) was actually due to an external factor (increased salience). When relative salience was controlled, the effect of T1-T3 lag disappeared, and with it the evidence for an $\mathrm{AB}$ for T3. Relative salience would also explain why, in red TTT triplets, identification accuracy for T3 is lower than for (the more salient) T1. 
Another factor that may plausibly account for the relative impairment of T3 can be found in the limitations of working memory storage capacity. It is generally assumed that this capacity is limited to about four items, give or take one or two, depending on individual differences (see, e.g., Cowan, 2001). The closer one gets to this memory limit, the more difficult it is for subsequent items to enter or stay in memory storage. This idea is nothing new, and has been demonstrated within the context of RSVP (see, e.g., Nieuwenstein \& Potter, 2006; Olivers et al., 2007; Reeves \& Sperling, 1986; Weichselgartner \& Sperling, 1987). By this reasoning, if $\mathrm{T} 1$ is more salient, it is more likely to be encoded into memory, thereby filling a memory slot and reducing the space available for trailing items, notably T3. In other words, the final target (T3) in a uniform three-target (TTT) condition is expected a priori to be at a slight disadvantage relative to the final target in a varied two-target (TDT) condition. This is why the highly accurate T3 performance in the uniform condition is so remarkable. Note that this account invokes capacity limitations, but of a kind rather different from those that have been proposed in resource depletion theories of the AB. Those theories state that $\mathrm{T} 1$ processing per se causes the $\mathrm{AB}$. Here, we state that it may be harder to remember additional information when memory is close to capacity. We do not believe, however, that memory limitations provide an account of the AB. This is because they do not explain the typically strong deficits found for two targets with an intervening distractor, relative to the sparing found when three targets are presented in succession.

Other evidence is also inconsistent with resource depletion accounts. For example, Kawahara et al. (2006), as well as Olivers et al. (2007), found that the AB, once fully induced, can be rapidly reversed: TDTT sequences revealed a pronounced $\mathrm{AB}$ for the second target, but hardly any for the third. Again, this is difficult to explain if the $\mathrm{AB}$ is held to be caused by resource depletion arising from T1 processing. Instead, we argue that the selection of targets from an RSVP stream is governed by a flexible control system that responds rapidly to online changes in the stream. When the system encounters a distractor, input is temporarily disrupted or inhibited. There is no need to invoke resource depletion.

\section{AUTHOR NOTE}

This work was supported by VIDI Grant 452-06-007 from NWO (the Netherlands Organization for Scientific Research) to C.N.L.O.; a Nissan Science Foundation grant to J.K.; a Canadian Foundation for Innovation New Opportunities grant and a British Columbia Knowledge Development Fund grant to T.M.S.; and Discovery grants from the Natural Sci- ences and Engineering Research Council of Canada to T.M.S. and V.D.L. Correspondence related to this article may be sent to C. N. L. Olivers, Department of Cognitive Psychology, Vrije Universiteit Amsterdam, Van der Boechorststr. 1, 1081 BT Amsterdam, The Netherlands (e-mail: cnl .olivers@psy.vu.nl).

\section{REFERENCES}

Chun, M. M., \& Potter, M. C. (1995). A two-stage model for multiple target detection in rapid serial visual presentation. Journal of Experimental Psychology: Human Perception \& Performance, 21, 109-127.

Cowan, N. (2001). The magical number 4 in short-term memory: A reconsideration of mental storage capacity. Behavioral \& Brain Sciences, 24, 87-185.

Di Lollo, V., Kawahara, J.-I., Ghorashi, S. M. S., \& EnNs, J. T. (2005). The attentional blink: Resource depletion or temporary loss of control? Psychological Research, 69, 191-200.

Dux, P. E., Asplund, C. L., \& Marois, R. (2008). An attentional blink for sequentially presented targets: Evidence in favor of resource depletion accounts. Psychonomic Bulletin \& Review, 15, 809-813.

Joliceur, P., \& Dell'Acqua, R. (1998). The demonstration of shortterm consolidation. Cognitive Psychology, 36, 138-202.

KaWahara, J.-I., Kumada, T., \& Di Lollo, V. (2006). The attentional blink is governed by a temporary loss of control. Psychonomic Bulletin \& Review, 13, 886-890.

Nieuwenstein, M. R., \& Potter, M. C. (2006). Temporal limits of selection and memory encoding: A comparison of whole versus partial report in rapid serial visual presentation. Psychological Science, 17, 471-475.

OLIVERS, C. N. L. (2007). The time course of attention: It's better than we thought. Current Directions in Psychological Science, 16, 11-15.

Olivers, C. N. L., van der Stigchel, S., \& Hulleman, J. (2007). Spreading the sparing: Against a limited-capacity account of the attentional blink. Psychological Research, 71, 126-139.

Potter, M. C., Staub, A., \& O'Connor, D. H. (2002). The time course of competition for attention: Attention is initially labile. Journal of Experimental Psychology: Human Perception \& Performance, 28, $1149-1162$.

Raymond, J. E., Shapiro, K. L., \& Arnell, K. M. (1992). Temporary suppression of visual processing in an RSVP task: An attentional blink? Journal of Experimental Psychology: Human Perception \& Performance, 18, 849-860.

Reeves, A., \& Sperling, G. (1986). Attention gating in short-term visual memory. Psychological Review, 93, 180-206.

Weichselgartner, E., \& Sperling, G. (1987). Dynamics of automatic and controlled visual attention. Science, 238, 778-780.

\section{NOTES}

1. We note that, although this analysis concerns a published data set, the comparisons we report here were not published before (nor could they have been derived, because in the previous study, T1 accuracy was reported collapsed across T1-T2 lags).

2. It deserves mentioning that the same pattern of results was also found when the white stimuli were brighter than the red (i.e., when the color luminances were not equalized).

(Manuscript received April 28, 2008; revision accepted for publication August 19, 2008.) 